\title{
Prioritizing investments in innovations to protect women from the leading causes of maternal death
}

\author{
Tara M Herrick*, Claudia M Harner-Jay, Alice M Levisay, Patricia S Coffey, Michael J Free and Paul D LaBarre
}

\begin{abstract}
PATH, an international nonprofit organization, assessed nearly 40 technologies for their potential to reduce maternal mortality from postpartum hemorrhage and preeclampsia and eclampsia in low-resource settings. The evaluation used a new Excel-based prioritization tool covering 22 criteria developed by PATH, the Maternal and Neonatal Directed Assessment of Technology (MANDATE) model, and consultations with experts. It identified five innovations with especially high potential: technologies to improve use of oxytocin, a uterine balloon tamponade, simplified dosing of magnesium sulfate, an improved proteinuria test, and better blood pressure measurement devices. Investments are needed to realize the potential of these technologies to reduce mortality.
\end{abstract}

Keywords: Maternal health, Postpartum hemorrhage, Preeclampsia, Eclampsia, Technology assessment, Oxytocin, Magnesium sulfate, Uterine balloon tamponade, Blood pressure measurement, Proteinuria test

\section{Background}

Innovations in health technologies and services are often least available to people with the greatest health needs $[1,2]$. Because many women in low-resource settings lack access to basic maternal health technologies and services, more than 30 women die each hour from complications related to pregnancy and childbirth, mostly in sub-Saharan Africa and South Asia [3,4].

Many factors make it difficult to get needed maternal health innovations to women in low-resource settings [1]. These include serious health system deficiencies, such as the limited capacity of health staff. They also include technology-focused challenges, such as:

- Complicated user requirements for technologies, especially for health workers without advanced training.

- Weak or nonexistent distribution systems.

- Disparate markets and small sales volumes that drive up costs for manufacturing and distribution.

- Expensive clinical studies.

- Restrictive policy and regulatory guidelines.
Targeted investment and collaboration to address these challenges may help to bring lifesaving technologies to all women rather than just those in the richest countries.

Millennium Development Goal 5 aims to reduce the maternal mortality ratio by 75 percent by $2015[3,4]$. To accelerate progress toward this goal, Merck \& Co., Inc., launched Merck for Mothers, a ten-year initiative to reduce maternal mortality.

In October 2011, PATH received funding through Merck for Mothers to identify the most promising technologies with the potential to help save women's lives. PATH is an international nonprofit organization with nearly four decades of experience adapting, developing, and introducing health technologies for developing countries.

\section{Methods}

PATH identified 38 technologies for evaluation, with emphasis on those that address postpartum hemorrhage and preeclampsia and eclampsia, the leading causes of maternal death. We identified these technologies by reviewing World Health Organization (WHO) guidelines, Bill \& Melinda Gates Foundation Grand Challenges in Global Health awards, and US Agency for International Development Saving Lives at Birth finalists, as well as by soliciting input from maternal health experts and other stakeholders.

\footnotetext{
* Correspondence: therrick@path.org

PATH, PO Box 900922, Seattle, WA 98109, USA
} 
We used a rigorous, multiphased approach to evaluate the technologies and then create strategies to advance those with the greatest promise. The goal of phase 1 was to identify the 10 best technology opportunities for additional analysis. To winnow the list, PATH needed a way to assess all the criteria that affect a technology's potential impact.

Because our review of the literature found that no existing assessment tool would meet our needs, PATH developed a strategic prioritization tool for the project [see Additional file 1]. The tool is a decision matrix consisting of 22 criteria, each representing a discrete element of the technology's value proposition and potential for impact (see Table 1). The matrix captures a score for each criterion, a cumulative score, and score substantiation for each technology. To develop the criteria and related definitions, project staff consulted internal maternal health experts, commercial firms, and other sources [5]. The tool and detailed information on the criteria, definitions, and process are available online at http://sites. path.org/mnhtech/assessment/tool/. (If the Uniform Resource Locator [URL], or web address, for a site mentioned in this manuscript changes, the authors will inform readers by adding a comment to the manuscript online).

The tool encompasses five categories of criteria that influence potential impact:

- The gap-filling potential for health. This category focuses on the technology's potential for reducing morbidity and mortality and filling existing "gaps." Technologies that are used earlier in the treatment continuum (e.g., prevention) generally receive a higher ranking because of their potential to prevent life-threatening conditions, reduce unnecessary morbidity and mortality, and reduce the need for emergency care. Other considerations in this category include the strength of available data and the percentage of cases that could be better managed with the technology.

- Technology performance. The criteria in this category concern the clinical performance, safety, and ease of use of the technology compared to an existing benchmark, which we identified by reviewing WHO guidelines. We also evaluated usage requirements to highlight factors that may influence appropriate use in low-resource settings. These requirements include fragile handling, shelf life, waste disposal, specialized storage needs, electrical power requirements, infection prevention, and training.

- Enabling factors. We use this category to determine whether an enabling environment is in place for successful technology introduction. Factors include alignment with international guidelines, existing support, an acceptability profile, and existing capabilities.

- Market considerations. We use the criteria in this category to evaluate the time and investment needed to bring a technology to market as well as the health care setting and type of health worker most likely to use the technology.

- Unique considerations. With this category, we assess other factors that may significantly affect the introduction and scaling of the technology, such as system and bundling requirements and potential disruption of existing systems. "Disruption" is defined as any significant shift in costs, roles, or risks of any of the stakeholders that are involved in the acquisition, distribution, or deployment of technologies.

In phase 2 of the assessment, PATH applied different methods to further narrow the focus to a handful of technologies with the greatest promise. For example, PATH used the Maternal and Neonatal Directed Assessment of Technology (MANDATE) model developed by Research Triangle Institute to assess the potential number of lives saved for each technology (for more information, see http://mnhtech.org). We also conducted an online survey with maternal health experts to rank candidate technologies for their potential to reduce mortality in sub-Saharan African and South Asia. The survey was sent to 63 maternal health experts with developingcountry expertise from a variety of organizations and was completed by 34 individuals ( $54 \%$ response rate) in April 2012.

\section{Results and discussion}

Our systematic evaluation identified five types of technologies with especially high potential for reducing deaths from postpartum hemorrhage and preeclampsia and eclampsia in low-resource settings:

- Technologies to facilitate appropriate use of oxytocin. Although WHO recommends oxytocin for preventing and managing postpartum hemorrhage, this drug is unavailable to many women, as highlighted in a recent report from the United Nations Commission on Life-Saving Commodities for Women and Children [6]. Expanding its use will likely require developing and introducing easy-touse formats appropriate for low-resource settings. Developing heat-stable formulations, for example, may eliminate or reduce the need for cold chain storage and enable broader use, especially for home births [7]. Also, formulations for alternative methods of administration (e.g., via inhalation or sublingual delivery) may increase safety and allow even 
Table 1 Assessment criteria for technologies that address postpartum hemorrhage and preeclampsia and eclampsia

\begin{tabular}{ll}
\hline Category & Criteria \\
\hline Gap & Gap-filling potential for health
\end{tabular}
Evaluation approach (high, medium, low)*

Evaluate what layers of the treatment continuum could most greatly reduce mortality (e.g., prevention, diagnostics, treatment), the strength of the available data, and the percentage of cases that could be managed

Technology performance Clinical evidence (efficacy/effectiveness)

Safety (patient/health care worker)

Ease of use

Usage requirements (e.g., durability, shelf life, electricity, storage temperature)

\section{Enabling factors}

Alignment with internationally recognized guidelines (e.g., the World Health Organization, the International Federation of Gynecology and Obstetrics, and the International Confederation of Midwives)

Donor financial support (product development or implementation)

Other nonfinancial support

Acceptability profile

Organizational capabilities

Market analysis

\section{Manufacturing costs}

Distribution system requirements (warehouse, cold chain, transportation factors)

Manufacturing plan established

Target setting (community, primary health care, hospital)

Target provider/administrator

Potential multiple markets (additional value to the health care system)

Technology readiness level (clinical/regulatory development)

Cost of clinical development

Clarity of regulatory/clinical pathways

Unique considerations System requirements (disruption)

Product bundling

Other
Superior to benchmark, ${ }^{* *}$ similar to benchmark, inferior to benchmark

Superior to benchmark,** similar to benchmark, inferior to
benchmark

Superior to benchmark, ${ }^{* *}$ similar to benchmark, inferior to benchmark

Technology has two or fewer usage requirements, technology has between three and five usage requirements, technology has greater than five usage requirements

Technology is recommended by at least one organization, divergent opinions exist, technology is not recommended

Funding greater than US\$5 M, between US\$1 M and US $\$ 5 \mathrm{M}$, less than US\$1 M (or no funding identified)

Placeholder for other nonfinancial supporters that may not be captured elsewhere; not scored

Broadly acceptable, mixed, acceptable in few geographies (South Asia and sub-Saharan Africa)

All key partners have experience with the technology, a subset of key partners has experience with the technology, none of the key partners has experience with the technology

Superior to benchmark** (lower cost), similar to benchmark, inferior to benchmark (higher cost)

Infrastructure for a distribution channel(s) exists and high likelihood of utilization, infrastructure for distribution channel(s) exists and moderate likelihood of utilization, distribution channel(s) does not exist or a low likelihood of utilization

Capture manufacturing information including delays, hurdles, risk, and complexity; not scored

Capture setting(s) where the technology would be used not scored Community health care worker, nurse or midwife, physician Additional uses identified (high), no additional uses (low)

Regulatory or commercialization, confirmatory, discovery, or exploratory

Less than US\$5 M, between US\$5 M and US\$50 M, greater than US\$50 M

Class I US Food and Drug Administration (USFDA) device, class II USFDA device, class III USFDA device or requires efficacy data (e.g., drugs)

Low system requirements (training/infrastructure), modest system requirements, high system requirements

No other technologies required for impact, likely need to bundle with one technology, likely need to bundle with two or more technologies

Placeholder for other unique considerations; not scored

\footnotetext{
"Detailed definitions are available at http://sites.path.org/mnhtech/assessment/tool/.

**Benchmark based on WHO treatment guidelines or the best benchmark for that technology category.
} 
lower-level health workers to give the medicine. There is a need for accelerated investment in new heat-stable formulations and alternative administration methods.

- Uterine balloon tamponade. This elastomeric bladder-like device can be inserted into the uterus and filled with saline to control severe postpartum bleeding [8]. It works rapidly and effectively, reducing the need for risky surgical interventions and blood transfusions $[9,10]$. Commercially available models, however, are too expensive for widespread use in developing countries, and performance of some versions is inconsistent. Investments are needed to gather clinical evidence for efficacy in low-resource settings through randomized, controlled trials of new, affordable devices under development.

- Simplified dosing of magnesium sulfate. WHO has identified magnesium sulfate as the most effective, safe, and low-cost medication for preventing and treating convulsions for women with severe preeclampsia or eclampsia [11]. Recommended dosing, however, requires a complicated combination of intravenous and intramuscular injection, and health workers in peripheral facilities may be unable to provide this treatment. Simplified dosing and dose packaging are feasible and could help providers more easily give women the medicine they need [12]. There is an immediate need for a randomized, controlled trial to determine whether a simplified, single, loading-dose-only regimen can effectively prevent and treat preeclampsia and eclampsia.

- Improved proteinuria test. Although detection of proteinuria is critical for diagnosing preeclampsia, most women in developing countries are not tested for proteinuria because tests are either unavailable or simply not used, and existing technologies have poor performance [13]. Investments are needed to assess novel, easy-to-use methods of proteinuria detection in the laboratory and then develop advanced prototypes for evaluation in lowresource settings.

- Blood pressure measurement devices. Like testing for proteinuria, measurement of blood pressure is critical for diagnosing preeclampsia. Health care providers in low-resource settings often fail to detect hypertension because suitable measurement devices are not available or they do not have needed skills [14]. There is a need for low-cost blood pressure measurement devices that are validated for use with pregnant women and that conform to WHO recommendations and international standards.
In phase 3 of the project, PATH created strategies to advance select technologies for introduction, scale-up, and ultimately impact.

\section{Conclusion}

Further development and introduction of maternal health technologies designed for use in low-resource settings will help to save many women's lives. Through a systematic assessment, PATH has identified five technologies with high potential for impact and provided a basis for prioritizing investments. Especially in times of significant resource constraints, it is critical for donors, national governments, businesses, and other stakeholders to determine the best use of scarce resources to improve maternal health. Investing in high-impact technologies and services and collaborating to advance their use will build brighter futures for women, families, communities, and nations.

\section{Additional file}

Additional file 1: Strategic prioritization tool.

\section{Abbreviations}

MANDATE: Maternal and neonatal directed assessment of technology PATH: Program for Appropriate Technology in Health; WHO: World Health Organization.

\section{Competing interests}

The authors declare that they have no competing interests.

\section{Authors' contributions}

All authors contributed substantially to the assessment underlying this article and to developing, reviewing, and editing the manuscript. All authors approved the final version.

\section{Acknowledgments}

The activities discussed in this publication were supported by funding from Merck through its Merck for Mothers Program. The content of this publication is solely the responsibility of the authors and does not represent the official views of Merck. We thank PATH staff member John Ballenot for providing writing and editing services. We also thank PATH staff members Susheela Engelbrecht and Sara Tifft for their valuable contributions to this project.

Received: 26 April 2013 Accepted: 3 January 2014

Published: 9 January 2014

\section{References}

1. Howett P, Darzi A, Yang G-Z, et al: Technologies for global health. Lancet 2012, 380:507-535.

2. Sinha SR, Barry M: Health technologies and innovation in the global health arena. N Engl J Med 2011, 365(9):779-782.

3. World Health Organization (WHO): Trends in Maternal Mortality: 1990 to 2010. Geneva: WHO; 2012.

4. Nations U: Millennium Development Goals Report 2012. New York: United Nations; 2012

5. Shelton JD: Twenty criteria to make the best of scarce health resources in developing countries. BMJ 2011, 343:d7023.

6. United Nations Commission on Life-Saving Commodities for Women and Children: Commissioners' Report September 2012. New York: United Nations; 2012.

7. MDG5 Meshwork for Improving Maternal Health: Public private partnership for the development of heat-stable oxytocin. 2010. Available at: http:// www.mdg5-meshwork.org/content/public-private-partnership-developmentheat-stable-oxytocin. Accessed 4 December 2012. 
8. World Health Organization (WHO): Guidelines for the Management of Postpartum Haemorrhage and Retained Placenta. Geneva: WHO; 2012. Available at: http://apps.who.int/iris/bitstream/10665/75411/1/ 9789241548502_eng.pdf. Accessed 7 December 2012

9. Doumouchtsis SK, Papageorghiou AT, Vernier C, Arulkumaran S: Management of postpartum hemorrhage by uterine balloon tamponade: prospective evaluation of effectiveness. Acta Obstet Gynecol Scand 2008, 87:849-855.

10. Tindell K, Garfinkel R, Abu-Haydar E, et al: Uterine balloon tamponade for the treatment of postpartum haemorrhage in resource-poor settings: a systematic review. BJOG 2012. doi:10.1111/j.1471-0528.2012.03454.x. [Epub ahead of print].

11. World Health Organization (WHO): WHO Recommendations for the Prevention and Treatment of Preeclampsia and Eclampsia. Geneva: WHO; 2011.

12. Dhakal G, Subedi M, Paudel K: Magnesium sulphate in management of severe pre-eclampsia and eclampsia. J Nepal Health Res Counc 2012, 10(21):113-117.

13. Osungbade KO, Ige OK: Public health perspectives of preeclampsia in developing countries: implication for health system strengthening. J Pregnancy 2011, 481095. doi: 10.1155/2011/481095.

14. Villar J, Say L, Shennan A, et al: Methodological and technical issues related to the diagnosis, screening, prevention, and treatment of preeclampsia and eclampsia. Int J Gynaecol Obstet 2004, 85:S28-S41.

doi:10.1186/1471-2393-14-10

Cite this article as: Herrick et al:: Prioritizing investments in innovations to protect women from the leading causes of maternal death. BMC

Pregnancy and Childbirth 2014 14:10.

\section{Submit your next manuscript to BioMed Central and take full advantage of:}

- Convenient online submission

- Thorough peer review

- No space constraints or color figure charges

- Immediate publication on acceptance

- Inclusion in PubMed, CAS, Scopus and Google Scholar

- Research which is freely available for redistribution 